\title{
Analysis of formulas to calculate the AC inductance of different configurations of nonmagnetic circular conductors
}

\author{
Francesca Capelli*, Jordi-Roger Riba**
}

*Electrical Engineering Department, Universitat Politècnica de Catalunya, Universitat Politècnica de Catalunya, 08222 Terrassa, Spain.

**Corresponding author: Electrical Engineering Department, Universitat Politècnica de Catalunya, 08700 Igualada, Spain, Tel.: +34 938035300; fax: +34 938031589. E-mail address: riba@ee.upc.edu (J.-R. Riba)

\begin{abstract}
The inductance of single- and multi-conductors intended for electronic devices, power transmission and distribution or grounding, lightning and bonding systems greatly depends on the specific geometry and the supply frequency. It is also influenced by skin and proximity effects. The inductance is an important design parameter, since it significantly influences the voltage drop in the conductor, thus raising reactive power consumption and limiting the conductors' current-carrying capacity. Although there exist some internationally accepted approximated and exact formulas to calculate the AC inductance of conductors in free air, its accuracy and applicability has been seldom analyzed in detail in the technical literature, which is done in this paper. Since such formulas can be used for a wide diversity of conductors' configurations and under different operating conditions, it is highly desirable to evaluate their applicability. This evaluation is carried out by comparing the results provided by the formulas with data provided by finite element method (FEM) simulation. The results provided in this paper prove that FEM results can be easily applied to a wide diversity of conductors' configurations and frequencies, thus being more general and often more accurate than the results provided by the formulas.
\end{abstract}

\section{KEYWORDS}

Inductance, finite element method, reactive power, voltage drop.

\section{INTRODUCTION}

Conductors supplied from an alternating current (AC) power source often present nonuniform current densities because of the induced eddy currents which cause the skin and proximity effects [1]-[4]. Under high-frequency supply, the electric current within a conductor is forced to flow within a small fraction of its cross section since eddy currents and their associated magnetic fields induced within the conductor alter the current distribution across the conductor. It is well known that the current distribution within a conductor has a great influence on its resistance and inductance [5]. As the power supply frequency is increased, the current within a conductor tends to crowd towards the outer surface [6], therefore increasing its effective resistance and decreasing the inner inductance. Both components of the conductor's impedance, that is, the reactance and the resistance generate voltage drops, which are especially important at high frequency operation, thus limiting the current-carrying capacity and increasing the operating temperature due to the active and reactive components of the electric power consumed by the conductor itself.

Inductance plays a key role in power cables and lines, as well as in lightning, grounding and bonding protection systems, especially at high-frequency operation. In the technical literature the change of the AC inductance in conductors has been less studied than the change of the AC resistance, probably because the inductance is highly influenced by the specific geometry of the analyzed problem. In lightning, grounding and bounding systems, the effects of the inductance are especially significant under the presence of high frequency impulse currents, since they generate a fast voltage drop along the conductor, thus hampering the impulse current to flow towards the grounding electrodes. In this case, due to the inductive effect, the grounding system is no longer equipotential [7]. This effect limits the effectiveness of the abovementioned protection systems used to avoid dangerous potential differences between different conductor objects in an electrical installation [8]. To ensure optimum performance, surge protection systems require a low inductance path to the ground. It is therefore very important to have a tool to accurately determine the inductance of the arrangement analyzed. It is also well-known that the overall impedance of a grounding electrode is greatly influenced by its geometry and earth resistivity, the analysis of the impact of these parameters not being covered by this paper.

The inductance highly relies on the area enclosed by the current path. In the case of an isolated conductor operated at high frequency, the current is forced to flow through the outer circumference of the conductor due to the skin effect [9]. Since the current is confined in a small fraction of the conductor's total cross section, its effective or AC resistance tends to increase whereas the effective internal inductance tends to decrease [10].

In the technical literature one can find both exact and simplified formulas to calculate the inductance in conductors, some of which are evaluated in this paper. Available analytical formulas cannot deal with effects such as non-circular cross-sectional 
geometries, multi-conductor systems or unbalanced multi-phase conductors among others. Unfortunately, due to the mathematical complexity involved, analytical formulas are only available for the simplest geometries [11], a limited range of conductors' dimensions and a restricted interval of frequencies since they often assume a constant current density across the conductor's cross section [12]. Most of the analyzed formulas assume that the conductors are placed in a homogenous medium and therefore they do not take into account other effects such as the influence of a conducting ground plane [13] or the proximity due to the presence of nearby conductors, otherwise inductance calculations would be more complex. Depending on the distance between the conducting ground and the analyzed conductors, system inductance [14] can be substantially reduced since according to the image theory, the ground effect may be modeled by equivalent conductors placed underneath the ground, through which flow a current in opposition to that of the source. As a consequence, it is required to apply numerical methods specifically intended for solving this type of problems, such as the finite element method (FEM) which can help in minimizing the abovementioned limitations. FEM simulations are well suited to deal with the application considered in this paper since they are very flexible and powerful and allow dealing with a wide range of geometries and frequencies even taking into account of eddy current effects [15]. It is internationally recognized that FEM simulations provide accurate solutions in problems involving power conductors, transformer windings or inductors [16], [17] where most of the formulas analyzed in this paper can provide inaccurate results due to the proximity effect, especially in transformers and rotating machines windings. This paper evaluates some of the formulas to determine the AC inductance of different conductors' shapes found in the technical literature and their accuracy is compared against results provided by FEM simulations. This paper is a continuation of the work presented in [3] in which the real part of the impedance of different conductors' configurations, that is the AC resistance, is analyzed in the $0 \mathrm{~Hz}-1$ $\mathrm{MHz}$ interval. This frequency range satisfies the quasi-stationary condition, so the displacement current can be neglected [9]. At the author's knowledge there are very few published scientific-technical works analyzing the applicability and accuracy of such formulas to calculate the $\mathrm{AC}$ inductance of different conductors' configurations, despite of the importance and the practical consequences of the inductance in conductors intended for power systems, thus this paper makes a contribution in this topic. It also proves that FEM simulations allow calculating the inductance in many conductors' configurations taking into account both skin and proximity effects, thus offering flexibility and accuracy, which in most configurations is not possible by means of analytical formulas.

\section{PARTIAL SELF-INDUCTANCE OF AN ISOLATED RECTILINEAR CONDUCTOR IN FREE AIR}

Inductance can be defined as the relationship between the magnetic flux through a surface bounded by a closed path and the current that generates this flux. Inductance is usually associated with loops or coils although a straight segment of a conductor or a portion of a loop also has its own inductance, which is known as partial inductance. In this case it is assumed that the straight segment forms a loop where the return segment is at infinity. The partial inductance of the conductor segment can be defined as the ratio between the magnetic flux generated by the conductor's current between this segment and infinity and the conductor's current [18]. The concept of partial inductance allows calculating, for example, the inductance of an infinitely long and isolated conductor [19] where there is no loop current. In this case, it is possible to calculate the expected voltage drop due to the partial inductance of the conductor.

Under AC supply, and especially at high frequency operation or for large cross sections [20], the current density in any isolated conductor placed in free air tends to be concentrated near the conductor's surface due to the eddy currents induced by the alternating magnetic field accompanying the current in the conductor [21]. This effect lessens the effective cross section, thus increasing the $\mathrm{AC}$ resistance and reducing its partial self-inductance. The reduction of the effective cross section of the conductor has important practical effects [22], including a higher voltage drop, a temperature increase and a decrease of the conductor's ampacity.

Since the current flowing within a conductor generates a magnetic flux in its surroundings, inside and outside of the conductor, the partial self-inductance of an isolated single conductor has two main components, that is, the internal and external inductances. The total inductance or partial self-inductance of the conductor is obtained by adding the internal and external inductances [11]. Whereas the internal inductance, which represents the smallest component [23] is due to the magnetic field distribution inside the conductor, the external inductance is due to the total current within the conductor, since it involves the magnetic field distribution outside the conductor.

The skin effect reduces the magnetic field inside the conductor, since it is confined in a smaller volume as the frequency increases, therefore decreasing the internal inductance of the conductor [22]. At very high frequency operation the total conductor's inductance is therefore almost equal to the external inductance [24].

The conductors dealt with in this section are supposed to be rectilinear with isotropic nonmagnetic material properties and uniform cross sections.

\section{Isolated rectilinear conductor of circular cross section in free air}

Since infinitely long isolated solid conductors exhibit the simplest geometry, there are exact formulas to determine their partial self-inductance considering the skin effect. It is well-known that the skin effect must be considered for large cross sections or at high frequency operation [16], that is, when the skin depth $\delta$ is small. The skin depth can be calculated as, 


$$
\delta=1 / \sqrt{\pi \cdot f \cdot \mu_{r} \cdot \mu_{0} \cdot \sigma}
$$

$\sigma$ being the electrical conductivity of the conductor's material, $\mu_{r}$ its relative permeability, $\mu_{0}$ the permeability of free space and $f$ the supply frequency.

The calculation of the partial self-inductance of an isolated infinitely long round conductor in free air is reasonably simple when assuming a uniform current distribution over the cross section of the conductor [25]. The partial self-inductance of the wire is calculated by means of the surface integral of the magnetic flux density of the conductor's segment considered between the center of the conductor and infinity. The symmetry of this geometry makes this calculation simple, since the magnetic field is symmetric along a circumference concentric with the conductor's symmetry axis. When neglecting the skin effect, that is, at very low frequency operation, the current density can be assumed uniform across the cross section of the conductor and the internal partial self-inductance per unit length $\left(L / l_{u}\right)$ of an infinitely long conductor becomes a constant,

$$
L_{i, d c} / l_{u}=\frac{\mu_{0}}{8 \pi} \quad[\mathrm{H} / \mathrm{m}]
$$

where $l_{u}$ is the unity length. It is worth noting that (2) is a constant, and thus independent of the conductor's radius. However, at high frequency operation, that is, when considering the skin effect, the calculation is more complex, and the following exact formula must be applied [26]-[28],

$$
L_{i} / l_{u}=\frac{4}{q} \cdot\left(L_{i, d c} / l_{u}\right) \cdot \frac{\operatorname{ber}(q) \cdot \operatorname{ber}^{\prime}(q)+\operatorname{bei}^{\prime}(q) \cdot b e i^{\prime}(q)}{\left[\operatorname{ber}^{\prime}(q)\right]^{2}+\left[\operatorname{bei}^{\prime}(q)\right]^{2}}[\mathrm{H} / \mathrm{m}]
$$

where $d_{c}$ is the diameter of the conductor, $q=d_{c} /(\sqrt{2} \delta)$, kind zero-order Kelvin functions [29], whereas bei' and ber' are their derivatives. The series expansion in the Kelvin functions can produce an arithmetic overflow of the computer capacity when $q$ is large, that is, for large conductor's cross sections or at very high frequency operation, when the skin depth is very small.

The external partial self-inductance of an infinitely long round conductor of radius $r$ can be calculated as [30],

$$
L_{e} / l_{u}=\frac{\mu_{0}}{2 \pi} \cdot \ln (R / r) \quad[\mathrm{H} / \mathrm{m}]
$$

$r$ being the radius of the conductor and $R$ the radius of the fictitious cylinder considered to calculate the external partial selfinductance, which is shown in Fig. 1.

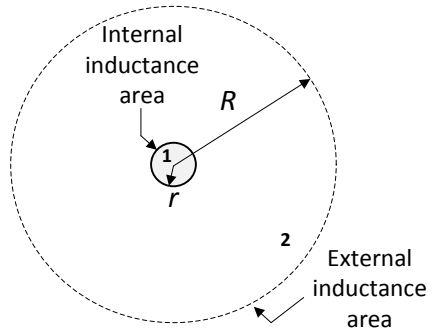

Fig. 1. Regions to calculate the internal and external inductances in an infinitely long round rectilinear conductor of radius $r$.

As shown in (4), the partial self-inductance depends on the arbitrary radius $R$ of the selected surface, which has no physical meaning since (4) neglects the effect of the return current, so in order to obtain the inductance of the conductor in a realistic way the complete circuit must be taken into account, including the return path [31].

As shown in Fig. 1, the internal partial self-inductance can be obtained from the magnetic energy calculated in region 1, whereas the external partial self-inductance in the region $r \leq$ radius $\leq R$ can be calculated from the stored magnetic energy in region 2. Finally, the total partial self-inductance of the conductor can be calculated by adding the internal and external inductances. It will be shown that the internal inductance is usually much smaller than the external inductance [32], this effect being more manifest at high frequency operation.

At low-frequency operation, Grigsby [30] approximates the total partial self-inductance per unit length of an infinitely long round conductor as,

$$
L_{t o t} / l_{u}=L_{i} / l_{u}+L_{e} / l_{u}=\frac{\mu_{0}}{8 \pi}+\frac{\mu_{0}}{2 \pi} \cdot \ln (R / r)=\frac{\mu_{0}}{8 \pi}+\frac{\mu_{0}}{2 \pi} \cdot \ln (R / r)=\frac{\mu_{0}}{2 \pi}\left[\ln \frac{R}{e^{-1 / 4} \cdot r}\right]=\frac{\mu_{0}}{2 \pi} \cdot \ln (R / G M R) \quad[\mathrm{H} / \mathrm{m}]
$$

GMR being the self-geometric mean radius [19], which is calculated as,

$$
G M R=\mathrm{e}^{-1 / 4} \cdot r=0.7788 \cdot r
$$

A formula valid when the conductor length is much larger than its radius, that is $l \gg r$, is provided in [33]-[35],

$$
L_{t o t} / l=\frac{\mu_{0}}{2 \pi} \cdot[\ln (2 \cdot l / r)-Y] \quad[\mathrm{H} / \mathrm{m}]
$$


where $Y=3 / 4$ for low frequency and $Y=1$ for high frequency applications. It shows that the increase of the conductor's radius has a limited effect on the high-frequency inductance.

According to Dengler [36], when taking into account complete skin effect, the partial self-inductance of a straight circular conductor of length $l$ can be calculated as,

$$
L_{t o t} / l=\frac{\mu_{0}}{4 \pi} \cdot\left\{2 \cdot\left[\ln \frac{2 \cdot l}{r}-1\right]+\frac{8 r}{\pi \cdot l}-\frac{r^{2}}{l^{2}}\right\} \quad[\mathrm{H} / \mathrm{m}]
$$

Wu et al. [37], approximate the partial self-inductance of straight segments of round cross section with radius $r$ and length $l$ by,

$$
L_{t o t} / l=\frac{\mu_{0}}{2 \pi} \cdot\left\{\ln \left[\frac{l}{r}+\sqrt{1+\frac{l^{2}}{r^{2}}}\right]-\sqrt{1+\frac{r^{2}}{l^{2}}}+\frac{r}{l}+\frac{1}{4}\right\}[\mathrm{H} / \mathrm{m}]
$$

\section{Isolated rectilinear tubular round conductor in free air}

Due of its geometry, a tubular round conductor allows minimizing weight and material costs compared to solid conductors, especially for large cross sections or at high frequency operation. By using tubular round conductors, the voltage drop due to the impedance of the conductors, power loss and reactive power can be significantly reduced. Fig. 2 shows the cross section of a round tubular conductor.

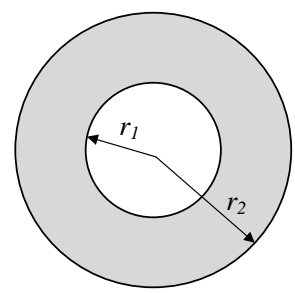

Fig. 2. Cross-section of a round tubular conductor

There exists an exact solution for calculating the per unit length internal partial self-inductance of an isolated infinitely long tubular conductor, which is based on the (ber, bei) and (ker, kei) functions, that is, the zero-order Kelvin functions of first kind and second kind, respectively and their derivatives (ber',bei') and (ker', kei'). When assuming that the current returns outside the conductor remotely it results in [26], [32],

$$
\frac{L_{i, a c}}{l_{u}}=\frac{\operatorname{imag}\left[\frac{j \cdot m}{2 \pi \sigma r_{2}} \cdot \frac{\operatorname{ber}\left(m \cdot r_{2}\right)+j \cdot b e i\left(m r_{2}\right)-C \cdot k e r\left(m \cdot r_{2}\right)-j C \cdot k e i\left(m \cdot r_{2}\right)}{\operatorname{ber}^{\prime}\left(m \cdot r_{2}\right)+j \cdot b e i^{\prime}\left(m r_{2}\right)-C \cdot k e r^{\prime}\left(m \cdot r_{2}\right)-j C \cdot k e i^{\prime}\left(m \cdot r_{2}\right)}\right]}{2 \pi f}[\mathrm{H} / \mathrm{m}]
$$

where $C=\frac{\operatorname{ber}^{\prime}(m \cdot \eta)+j \cdot b e i^{\prime}(m \eta)}{\operatorname{ker}^{\prime}(m \cdot \eta)+j k e i^{\prime}(m \cdot \eta)}, m=(2 \cdot \pi \cdot f \cdot \sigma)^{1 / 2}$, and $r_{1}$ and $r_{2}$ are, respectively, the inside and outside radius of the tubular conductor.

The DC internal partial self-inductance of the infinitely long tubular conductor with external return can be expressed as [2], [26],

$$
L_{i, d c} / l_{u}=\frac{\mu}{2 \pi}\left[\frac{r_{1}^{4}}{\left(r_{2}^{2}-r_{1}^{2}\right)^{2}} \cdot \ln \left(r_{2} / r_{1}\right)-\frac{3 r_{1}^{2}-r_{2}^{2}}{4\left(r_{2}^{2}-r_{1}^{2}\right)}\right][\mathrm{H} / \mathrm{m}]
$$

Similarly as in (4), the external inductance of an infinitely long tubular round conductor per unit length with outer radius $r_{2}$ can be calculated as [30],

$$
L_{e} / l_{u}=\frac{\mu_{0}}{2 \pi} \cdot \ln \left(R / r_{2}\right)[\mathrm{H} / \mathrm{m}]
$$

For infinitely long tubular conductors, Sapongin and Prokopenko [38] proposed the following formula to calculate the total partial self-inductance per unit length,

$$
\frac{L_{t o t}}{l_{u}}=\frac{\mu_{0}}{4 \pi}\left[\frac{1-\left(r_{1} / r_{2}\right)^{2}+2 \cdot\left(r_{1} / r_{2}\right)^{2} \cdot \ln \left(r_{1} / r_{2}\right)}{1-\left(r_{1} / r_{2}\right)^{2}}+2 \cdot \ln \left(R / r_{2}\right)\right][\mathrm{H} / \mathrm{m}]
$$

In the case of infinitely long thin-wall tubular conductors (13) becomes [38],

$$
L_{t o t} / l_{u}=\frac{\mu_{0}}{4 \pi} \cdot\left[t+t^{2} / 6-t^{4} / 30+2 \cdot \ln \left(R / r_{2}\right)\right][\mathrm{H} / \mathrm{m}]
$$

where $t=\left(r_{2}-r_{1}\right) / r_{2}<<1$. 


\section{INDUCTANCE OF A RETURN CIRCUIT OF RECTILINEAR CONDUCTORS IN FREE AIR}

As explained, when dealing with straight conductors, the effect of the return path must be considered, and then the dependence of $\mathrm{R}$ (radius of the external cylinder taken into account) in formulas such as (4), (5) and (12) disappears. In addition, the proximity effect arises, thus making more complex the derivation of analytical formulas.

\subsection{Return circuit of rectilinear round conductors}

Fig. 3 shows the geometry of a return circuit composed of two straight conductors of circular cross section, that is, with the currents flowing in opposite directions and with axial distance D.

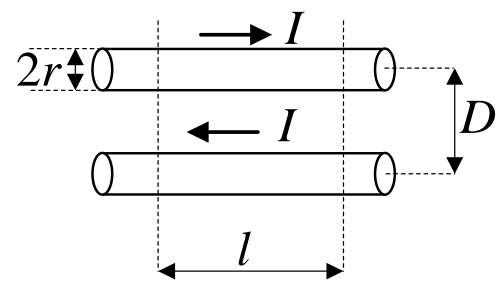

Fig. 3. Return circuit formed by two identical conductors of radius $r$ with the currents flowing in opposite directions.

The external inductance is associated to the magnetic flux surrounding the conductor, which stores magnetic energy, whereas the internal inductance neglects the external flux. The external partial inductance per unit length of a single infinitely long conductor under the supposition $D \gg r$ is given by [39],

$$
L_{e, 1 c} / l_{u}=\frac{\mu_{0}}{2 \pi} \cdot \ln \left(\frac{D}{r}\right)[\mathrm{H} / \mathrm{m}]
$$

$D$ being the distance between conductors' axes and $r$ the conductor's radius. Since in a return circuit there are two infinitely long conductors with currents flowing in opposite directions, the total partial external inductance per unit length is twice (15), that is,

$$
L_{e, 2 c} / l_{u}=\frac{\mu_{0}}{\pi} \cdot \ln \left(\frac{D}{r}\right)[\mathrm{H} / \mathrm{m}]
$$

The total partial inductance of two infinitely long parallel conductors with opposite currents takes into account both the internal inductance $L_{i, I_{c}} l_{l}$ given by (3) and the external inductance $L_{e, 2 c} d l_{u}$ given by (16), thus resulting in,

$$
L_{t o t, 2 c} / l_{u}=2 \cdot L_{i, 1 c} / l_{u}+L_{e, 2 c} / l_{u}=\frac{8}{q} \cdot\left(L_{i, d c} / l_{u}\right) \cdot \frac{\operatorname{ber}(q) \cdot \operatorname{ber}^{\prime}(q)+b e i(q) \cdot b e i^{\prime}(q)}{\left[\operatorname{ber}^{\prime}(q)\right]^{2}+\left[\operatorname{bei}^{\prime}(q)\right]^{2}}+\frac{\mu_{0}}{\pi} \cdot \ln \left(\frac{D}{r}\right)[\mathrm{H} / \mathrm{m}]
$$

Since (17) is based on the exact solution of an infinitely long isolated conductor, it doesn't take into account the proximity effect, and therefore it is not an exact formula. It is worth noting that (17) is not found in the technical literature although it is straightforwardly obtained from (3) and (16).

According to Sakis [31], when neglecting the skin effect, the total partial inductance of the infinitely long single-phase conductor results in,

$$
L_{t o t, 2 c} / l_{u}=2 \cdot L_{i, 1 c} / l_{u}+L_{e, 2 c} / l_{u}=\frac{\mu_{0}}{4 \pi}+\frac{\mu_{0}}{\pi} \cdot \ln \left(\frac{D}{r}\right)=\frac{\mu_{0}}{\pi} \ln \left(\frac{D}{G M R}\right)[\mathrm{H} / \mathrm{m}]
$$

where $G M R=\mathrm{e}^{-1 / 4} \cdot r=0.7788 \cdot r$.

Grigsby and other authors [30] express the total inductance of a return circuit of infinitely long round conductors as,

$$
L_{t o t, 2 c} / l_{u}=\frac{\mu_{0}}{\pi} \cdot \ln \left(\frac{D-r}{G M R}\right)[\mathrm{H} / \mathrm{m}]
$$

When dealing with two or more conductors, there is an interaction among the magnetic fields of each conductor, thus appearing the concept of partial mutual inductance between two conductors, which is defined as the ratio between the flux linkage from one conductor to infinity to the current flowing in the other conductor. When considering a return circuit in which the currents in the two straight conductors flow in opposite directions, the partial self-inductance of the return circuit can be obtained from the partial self-inductances of the individual conductors and the partial mutual inductance as [34], [40],

According to Rosa [40], the total inductance of the return circuit of round conductors and finite length $l$ is given by,

$$
L_{\text {circuit }}=L_{\text {conducto }, 1}+L_{\text {conductor }, 2}-2 \cdot M
$$

$M$ being the partial mutual inductance. If the conductors are identical, that is, with same lengths and radii, (20) results in,

$$
L_{t o t, 2 c}=2 \cdot L_{1 c}-2 \cdot M
$$

where $L_{l c}$ is the partial self-inductance of each single conductor, which is calculated as in (7). The partial mutual inductance $M$ can be expressed as, 


$$
M / l=\frac{\mu_{0}}{2 \pi} \cdot\left[\ln \left(\frac{l+\sqrt{l^{2}+D^{2}}}{D}\right)-\frac{\sqrt{l^{2}+D^{2}}}{l}+\frac{D}{l}\right] \approx \frac{\mu_{0}}{2 \pi} \cdot\left[\ln \left(\frac{2 \cdot l}{D}\right)-1+D / l\right][\mathrm{H} / \mathrm{m}]
$$

The approximation applied in (22) is valid when $l>D$. By substituting (7) and (22) in (21) it results in,

$$
L_{t o t, 2 c} / l=\frac{\mu_{0}}{\pi} \cdot\left[\ln \left(\frac{D}{r}\right)+1 / 4-D / l\right][\mathrm{H} / \mathrm{m}]
$$

\subsection{Isolated tubular round conductor with internal return in free air}

In this section a tubular conductor with internal return is analyzed. Fig. 4 shows this geometry, with inner radius of the inner and outer conductors, $r_{1}$ and $r_{2}$, respectively.

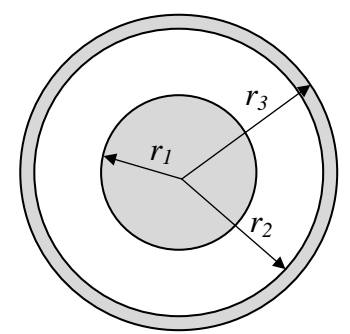

Fig. 4. Cross-section of a tubular conductor with inner return

According to Russell [41], when assuming that the current returns concentrically inside the conductor, the internal selfinductance per unit length is calculated as,

$$
\begin{aligned}
L_{i, 2 c} / l_{u}= & \frac{m}{4 \cdot \pi^{2} \cdot \sigma \cdot r_{1} \cdot Y\left(m r_{1}\right)}\left[\operatorname{ber}\left(m r_{1}\right) \cdot b e r^{\prime}\left(m r_{1}\right)+\operatorname{bei}\left(m r_{1}\right) \cdot b e i^{\prime}\left(m r_{1}\right)\right]+\frac{m \cdot b e r^{\prime}\left(m r_{1}\right)}{4 \cdot \pi^{2} \cdot \sigma \cdot r_{1} \cdot Y\left(m r_{1}\right)}\left[M \cdot b e r\left(m r_{2}\right)+N \cdot b e i\left(m r_{2}\right)+O \cdot \operatorname{ker}\left(m r_{2}\right)+P \cdot k e i\left(m r_{2}\right)\right]+ \\
& +\frac{m \cdot b e i^{\prime}\left(m r_{1}\right)}{4 \cdot \pi^{2} \cdot \sigma \cdot r_{1} \cdot Y\left(m r_{1}\right)}\left[M \cdot b e i\left(m r_{2}\right)-N \cdot b e r\left(m r_{2}\right)+O \cdot k e i\left(m r_{2}\right)-P \cdot \operatorname{ker}\left(m r_{2}\right)\right]
\end{aligned}
$$

where,

$$
\begin{aligned}
m= & \left(2 \pi f \mu_{0} \sigma\right)^{1 / 2}, \quad S(m x)=b e r^{\prime}(m x) \cdot \operatorname{ker}^{\prime}(m x)+b e i^{\prime}(m x) \cdot k e i^{\prime}(m x), \\
T(m x)=b e i^{\prime}(m x) \cdot \operatorname{ker}^{\prime}(m x)-b e r^{\prime}(m x) \cdot k e i^{\prime}(m x), \quad Y(m x)=b e r^{\prime 2}(m x)+b e i^{\prime 2}(m x), & \\
M= & \frac{-O \cdot S\left(m r_{3}\right)+P \cdot T\left(m r_{3}\right)}{Y\left(m r_{3}\right)} \\
N= & \frac{-O \cdot T\left(m r_{3}\right)-P \cdot S\left(m r_{3}\right)}{Y\left(m r_{3}\right)} \\
O= & -\frac{\left(\eta_{1} / r_{2}\right) \cdot Y\left(m r_{3}\right) \cdot\left[Y\left(m r_{3}\right) \cdot S\left(m r_{2}\right)-Y\left(m r_{2}\right) \cdot S\left(m r_{3}\right)\right] \cdot\left[b e i^{\prime}\left(m \eta_{1}\right) \cdot b e i^{\prime}\left(m r_{2}\right)+b e r^{\prime}\left(m \eta_{1}\right) \cdot b e r^{\prime}\left(m r_{2}\right)\right]}{\left[Y\left(m r_{3}\right) \cdot S\left(m r_{2}\right)-Y\left(m r_{2}\right) \cdot S\left(m r_{3}\right)\right]^{2}+\left[Y\left(m r_{2}\right) \cdot T\left(m r_{3}\right)-Y\left(m r_{3}\right) \cdot T\left(m r_{2}\right)\right]^{2}}+ \\
& +\frac{\left(\eta_{1} / r_{2}\right) \cdot Y\left(m r_{3}\right) \cdot\left[Y\left(m r_{2}\right) \cdot T\left(m r_{3}\right)-Y\left(m r_{3}\right) \cdot T\left(m r_{2}\right)\right] \cdot\left[b e r^{\prime}\left(m r_{1}\right) \cdot b e i^{\prime}\left(m r_{2}\right)-b e i^{\prime}\left(m \eta_{1}\right) \cdot b e r^{\prime}\left(m r_{2}\right)\right]}{\left[Y\left(m r_{3}\right) \cdot S\left(m r_{2}\right)-Y\left(m r_{2}\right) \cdot S\left(m r_{3}\right)\right]^{2}+\left[Y\left(m r_{2}\right) \cdot T\left(m r_{3}\right)-Y\left(m r_{3}\right) \cdot T\left(m r_{2}\right)\right]^{2}} \\
& =-\frac{\left(\eta_{1} / r_{2}\right) \cdot Y\left(m r_{3}\right) \cdot\left[Y\left(m r_{2}\right) \cdot T\left(m r_{3}\right)-Y\left(m r_{3}\right) \cdot T\left(m r_{2}\right)\right] \cdot\left[b e r^{\prime}\left(m \eta_{1}\right) \cdot b e r^{\prime}\left(m r_{2}\right)+b e i^{\prime}\left(m \eta_{1}\right) \cdot b e i^{\prime}\left(m r_{2}\right)\right]}{\left[Y\left(m r_{3}\right) \cdot S\left(m r_{2}\right)-Y\left(m r_{2}\right) \cdot S\left(m r_{3}\right)\right]^{2}+\left[Y\left(m r_{2}\right) \cdot T\left(m r_{3}\right)-Y\left(m r_{3}\right) \cdot T\left(m r_{2}\right)\right]^{2}}+ \\
& -\frac{\left(\eta_{1} / r_{2}\right) \cdot Y\left(m r_{3}\right) \cdot\left[Y\left(m r_{3}\right) \cdot S\left(m r_{2}\right)-Y\left(m r_{2}\right) \cdot S\left(m r_{3}\right)\right] \cdot\left[b e r^{\prime}\left(m \eta_{1}\right) \cdot b e i^{\prime}\left(m r_{2}\right)-b e i^{\prime}\left(m r_{1}\right) \cdot b e r^{\prime}\left(m r_{2}\right)\right]}{\left[Y\left(m r_{3}\right) \cdot S\left(m r_{2}\right)-Y\left(m r_{2}\right) \cdot S\left(m r_{3}\right)\right]^{2}+\left[Y\left(m r_{2}\right) \cdot T\left(m r_{3}\right)-Y\left(m r_{3}\right) \cdot T\left(m r_{2}\right)\right]^{2}}
\end{aligned}
$$

Although (24) considers the skin effect, it ignores the proximity effect between the conductors.

The DC per unit length internal inductance of the tubular conductor with internal return can be expressed as [26],

$$
L_{i, 2 c . d c} / l_{u}=\frac{\mu_{0}}{2 \pi}\left[\frac{r_{2}^{4}}{\left(r_{2}^{2}-r_{1}^{2}\right)^{2}} \cdot \ln \left(r_{2} / \eta_{1}\right)-\frac{3 r_{2}^{2}-r_{1}^{2}}{4\left(r_{2}^{2}-r_{1}^{2}\right)}\right][\mathrm{H} / \mathrm{m}]
$$

The per unit length external inductance of an infinitely long round coaxial conductor can be calculated as [34],

$$
L_{e, 2 c} / l_{u}=\frac{\mu_{0}}{2 \pi} \cdot \ln \left(\frac{r_{2}}{\eta_{1}}\right) \quad[\mathrm{H} / \mathrm{m}]
$$

Finally, when considering the skin effect, the total self-inductance can be calculated by adding (24) and (26).

When ignoring the skin effect, the total self-inductance per unit length can be calculated by adding the inner self-inductance of the round conductor which is given by (2), and the external self-inductance given by (26), thus resulting, 


$$
L_{t o t, 2 c} / l_{u}=\frac{\mu_{0}}{2 \pi} \cdot\left[\frac{1}{4}+\ln \left(\frac{m_{2}}{\eta_{1}}\right)\right] \quad[\mathrm{H} / \mathrm{m}]
$$

\section{RESULTS}

This section compares the results provided by the formulas developed in Sections II and III with those provided by twodimensional FEM simulations. Despite the important practical consequences of the inductance in conductor systems, there are virtually no published experimental data, therefore for comparison purposes FEM is taken as the reference method due to the accurate results provided [3]. To this end, the two-dimensional FEMM package [42] has been used since it is easy-to-use and provides fast and accurate solutions. Further details about the mathematical background of the FEMM formulation can be found in $[3]$.

All configurations studied in this section studied are analyzed by means of 2D-FEM models imposing a circular air perimeter with a Dirichlet boundary condition that forces the vector potential $A$ to be zero at the outer boundary. FEM-based simulations require discretizing the analyzed geometric domains, so they are divided into small elements. The size of such elements depends on the dimensions of the studied conductors and the analyzed frequency. Solutions provided by the FEM simulations proposed in this work have low computational requirements, thus providing fast results.

The inductance can be obtained from FEM simulations since in the case of linear magnetic regions, FEM calculates the stored magnetic energy as the volume integral [25],

$$
W_{\text {mag }}=\frac{1}{2} \iiint_{v} B \cdot H d v
$$

When dealing with an isolated rectilinear conductor, its partial inductance can be calculated from the stored magnetic energy as,

$$
W_{\text {mag }}=\int_{0}^{i} L \cdot i \cdot d i=\frac{1}{2} \cdot L \cdot i^{2}
$$

Therefore from (28) and (29) the conductor's partial self-inductance can be calculated as,

$$
L=2 \cdot W_{\text {mag }} / i^{2}[\mathrm{H}]
$$

The analyzed 2D-FEM domains involving straight conductors have a depth of one meter, so by applying (30) the inductance per unit length is directly calculated. Therefore it is straightforward to generalize the results to other conductors' lengths.

\subsection{AC inductance of an isolated solid round conductor in free air}

Many applications such as grounding systems, electronics, communication devices and power systems among others use round solid conductors [21], so it is of great interest to determine the change of the inductance as a function of the frequency.

Table I shows the evolution of the partial self-inductance predicted by FEM simulations and analytical formulas in the range 0 $\mathrm{Hz}-1 \mathrm{MHz}$ for a copper round conductor with diameter $39.28 \mathrm{~mm}$. Results from Table I show that the accuracy of (7), (8) and (9) greatly depends on the conductor length considered, so that the accuracy increases with the length of the conductor since for large conductor lengths the inductance per unit length provided by such formulas is very close to the exact one, with errors in the range 1.1-3.3\%. Another source of error is the skin effect. Table I clearly shows that (5), (7), (8) and (9) do not consider the skin effect because the results they provide are independent of the frequency although this effect is much less than that of the finite length of the conductor.

TABLE I

TOTAL PARTIAL SELF-INDUCTANCES PER UNIT LENGTH OBTAINED FROM EXACT AND APPROXIMATED ANALYTICAL FORMULAS AND FEM SIMULATIONS OF A COPPER ROUND SOLID CONDUCTOR WITH DIAMETER 39.28 MM $\left(1212 \mathrm{MM}^{2}\right)$.

\begin{tabular}{cccccc}
\hline \hline \multicolumn{5}{c}{ Total partial self-inductance per unit length } \\
\multicolumn{5}{c}{$\boldsymbol{L}_{\text {tot }} \boldsymbol{l}\left(\mathbf{( x 1 0} \mathbf{6}^{-\mathbf{6}} \mathbf{H} \mathbf{m}\right)$} \\
\hline $\begin{array}{c}\text { Frequency } \\
(\mathrm{Hz})\end{array}$ & Exact & $\mathrm{FEM}^{1}$ & $(5)^{1,2}$ & $\begin{array}{c}(7) \approx(8)^{2} \\
l=1-5000 \mathrm{~m}\end{array}$ & $l=1-5000 \mathrm{~m}$ \\
\hline 0 & 2.540 & 2.532 & 2.540 & $0.725-2.428$ & $0.779-2.478$ \\
50 & 2.532 & 2.525 & 2.540 & $0.725-2.428$ & $0.779-2.478$ \\
100 & 2.522 & 2.515 & 2.540 & $0.725-2.428$ & $0.779-2.478$ \\
200 & 2.513 & 2.506 & 2.540 & $0.725-2.428$ & $0.779-2.478$ \\
500 & 2.504 & 2.497 & 2.540 & $0.725-2.428$ & $0.779-2.478$ \\
1000 & 2.500 & 2.493 & 2.540 & $0.725-2.428$ & $0.779-2.478$ \\
10000 & 2.493 & 2.486 & 2.540 & $0.725-2.428$ & $0.779-2.478$ \\
100000 & 2.490 & 2.483 & 2.540 & $0.725-2.428$ & $0.779-2.478$ \\
1000000 & Overflow & 2.483 & 2.540 & $0.725-2.428$ & $0.779-2.478$ \\
\hline MAPE & - & $\mathbf{0 . 3 \%}$ & $\mathbf{1 . 1 \%}$ & $\mathbf{7 1 . 1 - 3 . 3 \%}$ & $\mathbf{6 9 . 0 - 1 . 3 \%}$ \\
\hline \hline
\end{tabular}

The percentage error among the different calculation systems has been calculated by means of the mean absolute percentage 
error or MAPE. Note that in this case, the MAPE has been calculated with respect to the results provided by the exact formula as,

$$
M=\frac{1}{n} \cdot \sum_{i=1}^{n}\left|\frac{y_{i}-\hat{y}_{i}}{y_{i}}\right|
$$

$n$ being the number of samples or frequencies calculated, and $y_{i}$ and $\hat{y}_{i}$ being respectively, the reference value of $i$-th frequency component and the forecast value.

\subsection{AC inductance of an isolated tubular round conductor in free air}

Tubular round conductors are very applied in induction furnaces, outdoor substation busbars, particle accelerators or MRI devices among others. Table II shows the evolution of the partial self-inductance predicted by FEM simulations and analytical formulas in the range $0 \mathrm{~Hz}-1 \mathrm{MHz}$ for a copper tubular round conductor with inner and outer radius $5 \mathrm{~mm}$ and $19.64 \mathrm{~mm}$, respectively.

TABLE II

TOTAL PARTIAL SELF-INDUCTANCE PER UNIT LENGTH FROM EXACT AND APPROXIMATED ANALYTICAL FORMULAS AND FEM SIMULATIONS OF A COPPER ROUND TUBULAR CONDUCTOR WHEN THE CURRENT RETURNS OUTSIDE THE CONDUCTOR REMOTELY WITH R $1=5$ MM AND R $2=19.64$ MM.

\begin{tabular}{|c|c|c|c|c|}
\hline \multicolumn{5}{|c|}{$\begin{array}{l}\text { Total partial self-inductance per unit length } \\
L_{\text {tot }} / l\left(\mathbf{x 1 0}^{-6} \mathrm{H} / \mathrm{m}\right)\end{array}$} \\
\hline Frequency $(\mathrm{Hz})$ & $(10)+(12)^{1}$ & FEM $^{1}$ & $(13)^{1,2}$ & $(14)^{1,2}$ \\
\hline 0 & 2.534 & 2.527 & 2.571 & 2.572 \\
\hline 50 & 2.530 & 2.523 & 2.571 & 2.572 \\
\hline 100 & 2.522 & 2.515 & 2.571 & 2.572 \\
\hline 200 & 2.513 & 2.506 & 2.571 & 2.572 \\
\hline 500 & 2.504 & 2.497 & 2.571 & 2.572 \\
\hline 1000 & 2.500 & 2.493 & 2.571 & 2.572 \\
\hline 10000 & 2.489 & 2.486 & 2.571 & 2.572 \\
\hline 100000 & 2.490 & 2.483 & 2.571 & 2.572 \\
\hline 1000000 & 2.490 & 2.483 & 2.571 & 2.572 \\
\hline MAPE & - & $0.3 \%$ & $2.5 \%$ & $2.6 \%$ \\
\hline
\end{tabular}

${ }^{1}$ Assume infinitely long conductors

${ }^{2}$ Neglect the skin effect

As shown in Table II, FEM results are more accurate than those obtained with analytical formulas since they also have into account the change in inductance due to the skin effect. It should be highlighted that results provided by (13) and (14) are independent of the conductor length.

\subsection{AC inductance of a return circuit of circular cross section in free air}

Return circuits composed of two conductors of circular cross section are of great interest since they have a wide range of applications. Tables III and IV show the change of the total partial inductance predicted by FEM simulations and analytical formulas in the range $0 \mathrm{~Hz}-1 \mathrm{MHz}$ for a return circuit of circular cross section of $100 \mathrm{~mm}^{2}$ spaced axially $100 \mathrm{~mm}$ and $500 \mathrm{~mm}$, respectively.

TABLE III

TOTAL PARTIAL INDUCTANCE PER UNIT LENGTH FROM APPROXIMATED ANALYTICAL FORMULAS AND FEM SIMULATIONS OF A RETURN CIRCUIT COMPOSED OF TWO ROUND CONDUCTORS $\left(\mathrm{D}=100 \mathrm{MM}, \mathrm{R}=5.642 \mathrm{MM}, \mathrm{A}=100 \mathrm{MM}^{2}\right)$.

\begin{tabular}{|c|c|c|c|c|c|}
\hline \multicolumn{6}{|c|}{$\begin{array}{c}\text { Total partial inductance per unit length } \\
L_{t o t} /\left(\mathbf{x}_{10}^{-6} \mathrm{H} / \mathrm{m}\right)\end{array}$} \\
\hline $\begin{array}{l}\text { Frequency } \\
(\mathrm{Hz})\end{array}$ & FEM $^{1}$ & $(17)^{1,3}$ & $(18)^{1,2}$ & $(19)^{1,2}$ & $\begin{array}{l}(23)^{2} \\
l=1-5000 \mathrm{~m}\end{array}$ \\
\hline 0 & 1.250 & 1.250 & 1.250 & 1.227 & $1.153-1.156$ \\
\hline 50 & 1.250 & 1.250 & 1.250 & 1.227 & $1.153-1.156$ \\
\hline 100 & 1.249 & 1.249 & 1.250 & 1.227 & $1.153-1.156$ \\
\hline 200 & 1.248 & 1.248 & 1.250 & 1.227 & $1.153-1.156$ \\
\hline 500 & 1.238 & 1.239 & 1.250 & 1.227 & $1.153-1.156$ \\
\hline 1000 & 1.220 & 1.221 & 1.250 & 1.227 & $1.153-1.156$ \\
\hline 10000 & 1.172 & 1.173 & 1.250 & 1.227 & $1.153-1.156$ \\
\hline 100000 & 1.154 & 1.157 & 1.250 & 1.227 & $1.153-1.156$ \\
\hline 1000000 & 1.148 & 1.150 & 1.250 & 1.227 & $1.153-1.156$ \\
\hline MAPE & - & $0.1 \%$ & $3.1 \%$ & $2.9 \%$ & $5.0-4.9 \%$ \\
\hline
\end{tabular}

TABLE IV

TOTAL PARTIAL INDUCTANCE PER UNIT LENGTH FROM APPROXIMATED ANALYTICAL FORMULAS AND FEM SIMULATIONS OF A RETURN CIRCUIT COMPOSED OF TWO ROUND CONDUCTORS ( $\mathrm{D}=500 \mathrm{MM}, \mathrm{R}=5.642 \mathrm{MM}, \mathrm{A}=100 \mathrm{MM} 2)$. 


\begin{tabular}{cllllc}
\hline \hline \multicolumn{7}{c}{$\boldsymbol{L}_{\text {tot }} \boldsymbol{l}\left(\mathbf{x 1 0}^{\mathbf{- 6}} \mathbf{H} / \mathbf{m}\right)$} \\
\hline $\begin{array}{c}\text { Frequency } \\
(\mathrm{Hz})\end{array}$ & $\mathrm{FEM}^{1}$ & $(17)^{1,3}$ & $(18)^{1,2}$ & $(19)^{1,2}$ & $\begin{array}{c}(23)^{2} \\
l=1-5000 \mathrm{~m}\end{array}$ \\
\hline 0 & 1.894 & 1.894 & 1.894 & 1.889 & $1.793-1.795$ \\
50 & 1.894 & 1.894 & 1.894 & 1.889 & $1.793-1.795$ \\
100 & 1.893 & 1.893 & 1.894 & 1.889 & $1.793-1.795$ \\
200 & 1.891 & 1.892 & 1.894 & 1.889 & $1.793-1.795$ \\
500 & 1.882 & 1.883 & 1.894 & 1.889 & $1.793-1.795$ \\
1000 & 1.864 & 1.865 & 1.894 & 1.889 & $1.793-1.795$ \\
10000 & 1.817 & 1.817 & 1.894 & 1.889 & $1.793-1.795$ \\
100000 & 1.799 & 1.801 & 1.894 & 1.889 & $1.793-1.795$ \\
1000000 & 1.793 & 1.794 & 1.894 & 1.889 & $1.793-1.795$ \\
\hline MAPE & - & $<0.1 \%$ & $\mathbf{2 . 0 \%}$ & $\mathbf{1 . 9 \%}$ & $\mathbf{3 . 5 - 3 . 4 \%}$ \\
\hline \hline
\end{tabular}

As shown in Tables III and IV, when the axial distance between conductors increases, the MAPE also decreases because the proximity effect is less pronounced. It is worth noting that whereas the results from (23) are almost independent of the conductor length, results from (17), (18) and (19) are completely independent of such variable. Another source of error is due to the eddy currents effect. As summarized in Tables III and IV, the results provided by (18), (19) and (23) are independent of the frequency since they do not consider the eddy currents effect.

\subsection{Inductance of a return circuit of a tubular conductor with internal return in free air}

Coaxial conductors are widely used in many applications including communication systems, television or radar among others. Tables V and VI show the change of the total partial inductance predicted by FEM simulations and analytical formulas in the range $0 \mathrm{~Hz}-1 \mathrm{MHz}$ for a return circuit of a tubular conductor with internal return for two configurations. The configuration analyzed in Table $\mathrm{V}$ consists of a copper coaxial conductor with inner return and $r_{1}=5 \mathrm{~mm}, r_{2}=10 \mathrm{~mm}$ and $r_{3}=12 \mathrm{~mm}$ (see Fig. 4), whereas the configuration analyzed in Table VI corresponds to a copper coaxial conductor with inner return and $r_{l}=10 \mathrm{~mm}$, $r_{2}=15 \mathrm{~mm}$ and $r_{3}=17 \mathrm{~mm}$. These two specific configurations are analyzed to determine the influence of the external inductance term, which depends on the $r_{2} / r_{1}$ ratio.

TABLE V

TOTAL PARTIAL INDUCTANCE PER UNIT LENGTH OBTAINED FROM APPROXIMATED ANALYTICAL FORMULAS AND FEM SIMULATIONS OF A COPPER COAXIAL CONDUCTOR WITH INNER RETURN AND $R_{1}=5 \mathrm{MM}, \mathrm{R}_{2}=10 \mathrm{MM}$ AND $\mathrm{R}_{3}=12 \mathrm{MM}\left(\mathrm{R}_{2} / \mathrm{R}_{1}=2\right)$.

\begin{tabular}{|c|c|c|c|c|}
\hline \multicolumn{5}{|c|}{$\begin{array}{c}\text { Total partial inductance per unit length } \\
\qquad L_{t o t} /\left(\mathbf{x}_{10}^{-7} \mathrm{H} / \mathrm{m}\right)\end{array}$} \\
\hline $\begin{array}{l}\text { Frequency } \\
(\mathrm{Hz})\end{array}$ & $\mathrm{FEM}^{1}$ & $(24)+(26)^{1,3}$ & $(25)+(26)^{1,2}$ & $(27)^{1,2}$ \\
\hline 0 & 2.019 & 2.019 & 2.018 & 1.886 \\
\hline 50 & 2.019 & 2.019 & 2.018 & 1.886 \\
\hline 100 & 2.017 & 2.017 & 2.018 & 1.886 \\
\hline 200 & 2.012 & 2.012 & 2.018 & 1.886 \\
\hline 500 & 1.981 & 1.981 & 2.018 & 1.886 \\
\hline 1000 & 1.909 & 1.909 & 2.018 & 1.886 \\
\hline 10000 & 1.584 & 1.452 & 2.018 & 1.886 \\
\hline 100000 & 1.449 & 1.388 & 2.018 & 1.886 \\
\hline 1000000 & 1.405 & Overflow & 2.018 & 1.886 \\
\hline MAPE & - & $1.57 \%$ & $13.15 \%$ & $12.82 \%$ \\
\hline
\end{tabular}

TABLE VI

TOTAL PARTIAL INDUCTANCE PER UNIT LENGTH OBTAINED FROM APPROXIMATED ANALYTICAL FORMULAS AND FEM SIMULATIONS OF A COPPER COAXIAL CONDUCTOR WITH INNER RETURN AND R1 $=10 \mathrm{MM}$ AND R2 $=15 \mathrm{MM}$ AND R $\mathrm{R}_{3}=17 \mathrm{MM}\left(\mathrm{R}_{2} / \mathrm{R}_{1}=1.5\right)$.

\begin{tabular}{|c|c|c|c|c|}
\hline \multicolumn{5}{|c|}{$\begin{array}{l}\text { Total partial inductance per unit length } \\
\qquad L_{\text {too }} l\left(\mathrm{x}^{-7} 0^{-7} \mathrm{H} / \mathrm{m}\right)\end{array}$} \\
\hline $\begin{array}{l}\text { Frequency } \\
(\mathrm{Hz})\end{array}$ & FEM $^{1}$ & $(24)+(26)^{1,3}$ & $(25)+(26)^{1,2}$ & $(27)^{1,2}$ \\
\hline 0 & 1.400 & 1.400 & 1.138 & 1.311 \\
\hline 50 & 1.393 & 1.393 & 1.138 & 1.311 \\
\hline 100 & 1.375 & 1.375 & 1.138 & 1.311 \\
\hline 200 & 1.320 & 1.320 & 1.138 & 1.311 \\
\hline 500 & 1.189 & 1.189 & 1.138 & 1.311 \\
\hline 1000 & 1.105 & 1.105 & 1.138 & 1.311 \\
\hline 10000 & 0.921 & 0.874 & 1.138 & 1.311 \\
\hline
\end{tabular}




\begin{tabular}{ccccc}
100000 & 0.846 & 0.811 & 1.138 & 1.311 \\
1000000 & 0.821 & Overflow & 1.138 & 1.311 \\
\hline MAPE & $\mathbf{-}$ & $\mathbf{1 . 1 \%}$ & $\mathbf{1 9 . 1 \%}$ & $\mathbf{2 2 . 6 \%}$ \\
\hline \hline
\end{tabular}

${ }^{1}$ Assume infinitely long conductors

${ }^{2}$ Neglect the skin and proximity effects

${ }^{3}$ Neglect the proximity effect

Results from Tables V and VI clearly show that the accuracy of (24) + (26), (25) + (26) and (27) greatly depends on the specific configuration. The main contribution in the total inductance is due to the external inductance term given by (26), which is highly influenced by the ratio $r_{2} / r_{l}$. Therefore the configuration analyzed in Table $\mathrm{V}$ shows a higher inductance than that in Table VI because the first configuration has a higher ratio $r_{2} / r_{1}$. As deduced from Tables V and VI, for frequencies $10 \mathrm{kHz}$ and above the proximity effect becomes more important and thus the difference between FEM results and those attained by applying $(24)+(26)$ is not negligible.

The 2D meshes of the conductors' configurations analyzed in this section through FEM simulations consist of a variable number of 2D triangular element, which depends on the geometry and dimensions of the problem and the frequency analyzed. The meshes of the geometries analyzed in Tables V and VI consist of a variable number of triangular elements within $360000(0$ $\mathrm{Hz})$ and $840000(1 \mathrm{MHz})$. The computational time required to perform such simulations was between $20 \mathrm{~s}$ and $90 \mathrm{~s}$ when using a computer with an Intel Xeon CPU, E5-2626 processors and 32 GB of RAM memory.

\section{CONCLUSION}

This paper has analyzed the accuracy of different exact and approximate formulas to calculate the inductance of different conductors' configurations carrying AC currents in a broad frequency interval. The results provided by these formulas have been compared with those obtained through two-dimensional FEM simulations, which are used as the reference method due to its accuracy and flexibility. It has been shown that analytical exact formulas are only available for the simplest and symmetrical geometries, and that when this symmetry is lost or when proximity effects arise, the accuracy of such formulas is highly affected. Results attained have shown the limitations of the commonly-used analyzed formulas in the frequency range comprising $0 \mathrm{~Hz}$ and $1 \mathrm{MHz}$, and have shown which specific configurations are more suitable to minimize the total inductance.

\section{ACKNOWLEDGMENTS}

The authors would like to thank the Spanish Ministry of Economy and Competitiveness and Generalitat de Catalunya for the financial support received under project RTC-2014-2862-3 and Doctorat Industrial under project DI-024-2013, respectively.

\section{REFERENCES}

[1] W. I. Middleton and E. W. Davis, "Skin effect in large stranded conductors at low frequencies," J. Am. Inst. Electr. Eng., vol. 40, no. 9, pp. 757-763, Sep. 1921.

[2] V. T. Morgan, "The Current Distribution, Resistance and Internal Inductance of Linear Power System Conductors-A Review of Explicit Equations," IEEE Trans. Power Deliv., vol. 28, no. 3, pp. 1252-1262, Jul. 2013.

[3] J.-R. Riba, “Analysis of formulas to calculate the AC resistance of different conductors' configurations," Electr. Power Syst. Res., vol. 127, pp. 93-100, Oct. 2015.

[4] J.-R. Riba, "Calculation of the ac to de resistance ratio of conductive nonmagnetic straight conductors by applying FEM simulations," Eur. J. Phys., vol. 36, no. July, pp. 1-10, 2015.

[5] L. Cohen, "The influence of frequency on the resistance and inductance of solenoidal coils," Dolezalek, Ann. der Phys., vol. 12, pp. $161-178,1903$.

[6] A. W. Barr, "Calculation of Frequency-Dependent Impedance for Conductors of Rectangular Cross Section," AMP J. Technol., vol. 1, pp. 91-100, 1991.

[7] J. He, R. Zeng, and B. Zhang, Methodology and Technology for Power System Grounding - Jinliang He, Rong Zeng, Bo Zhang. Singapore: John Wiley \& Sons Singapore Pte. Ltd., 2013.

[8] Eritech, "Grounding \& Bonding For Electrical Systems." p. 70, 2015.

[9] O. Coufal, "Current density in two solid parallel conductors and their impedance," Electr. Eng., vol. 96, no. 3, pp. 287-297, Sep. 2014.

[10] E. B. Joffe and K.-S. Lock, Grounds for Grounding: A Circuit-to-System Handbook. Hoboken, New Jersey: John Wiley \& Sons, Inc, 2010.

[11] P. Patel, "Calculation of Total Inductance of a Straight Conductor of Finite Length,” Phys. Educ., vol. July - Sep, pp. 193-198, 2009.

[12] O. Coufal, "On Resistance and Inductance of Solid Conductors," J. Eng., vol. 2013, pp. 1-14, 2013.

[13] X. Jia, L. Liu, and G. Fang, “The Finite-Conducting Ground's Effect on the Inductance of a Rectangular Loop,” J. Sensors, vol. 2016, pp. 1-11, 2016.

[14] J.-L. Schanen, C. Guerin, J. Roudet, and G. Meunier, "Influence of a conductive plane on loop inductance," IEEE Trans. Magn., vol. 31, no. 3, pp. 2127-2130, May 1995.

[15] R. Sitar, Ž. Štih, and Z. Valković, "Experimental verification of quasi-nonlinear modeling of magnetic steel for time-harmonic eddy current loss calculation," Electr. Eng., pp. 1-7, May 2016.

[16] U. Patel and P. Triverio, "Accurate Impedance Calculation for Underground and Submarine Power Cables using MoM-SO and a Multilayer Ground Model,” IEEE Trans. Power Deliv., pp. 1-1, 2015. 
[17] F. de Leon, S. Jazebi, and A. Farazmand, "Accurate Measurement of the Air-Core Inductance of Iron-Core Transformers With a NonIdeal Low-Power Rectifier," IEEE Trans. Power Deliv., vol. 29, no. 1, pp. 294-296, Feb. 2014.

[18] C. R. Paul, "What is 'partial inductance'?," in 2008 IEEE International Symposium on Electromagnetic Compatibility, 2008, pp. 1-23.

[19] H. A. Aebischer and B. Aebischer, "Improved Formulae for the Inductance of Straight Wires," Advanced Electromagnetics, vol. 3, no. 1. pp. 31-43, 08-Sep-2014.

[20] U. S. Gudmundsdottir, "Proximity effect in fast transient simulations of an underground transmission cable," Electr. Power Syst. Res., vol. 115, pp. 50-56, Oct. 2014.

[21] M. M. Al-Asadi, A. P. Duffy, A. J. Willis, K. Hodge, and T. M. Benson, "A simple formula for calculating the frequency-dependent resistance of a round wire," Microw. Opt. Technol. Lett., vol. 19, no. 2, pp. 84-87, Oct. 1998.

[22] G. S. Smith, "A simple derivation for the skin effect in a round wire," Eur. J. Phys., vol. 35, no. 2, p. 025002 , Mar. 2014.

[23] Z. Piatek, B. Baron, T. Szczegilniak, D. Kusiak, and A. Pasierbek, "Self inductance of long conductor of rectangular cross section," Prz. Elektrotechnikzny (Electrical Rev., vol. 88, no. 8, pp. 323-326, 2012.

[24] G. O. Ludwig and M. C. R. Andrade, "External inductance of large to ultralow aspect-ratio tokamak plasmas," Phys. Plasmas, vol. 5, no. 6, p. 2274, Jun. 1998.

[25] C. R. Paul, Inductance: Loop and Partial. Hoboken, New Jersey: John Wiley \& Sons, Inc, 2010.

[26] W. Mingli and F. Yu, "Numerical calculations of internal impedance of solid and tubular cylindrical conductors under large parameters," IEE Proc. - Gener. Transm. Distrib., vol. 151, no. 1, p. 67, 2004.

[27] S. Ramo, J. R. Whinnery, and T. Van Duzer, Fields and Waves in Communication Electronics, 3rd Editio. John Wiley \& Sons Inc., 1994.

[28] S. A. Schelkunoff, "The Electromagnetic Theory of Coaxial Transmission Lines and Cylindrical Shields," Bell Syst. Tech. J., vol. 13, no. 4, pp. 532-579, Oct. 1934.

[29] N. W. McLachlan, Bessel Functions for Engineers. Clarendon Press, 1961.

[30] L. Grigsby, Electric power generation, transmission, and distribution, Third. Boca Raton, FL, FL: CRC Press, Taylor \& Francis Group, 2012.

[31] A.P. Sakis Meliopoulis, Power System Grounding and Transients: An Introduction. New York: Marcel Dekker Inc., 1988.

[32] N. D. Tleis, Power Systems Modelling and Fault Analysis. Elsevier, 2008.

[33] S. Caniggia and F. Maradei, Signal Integrity and Radiated Emission of High-Speed Digital Systems. Chicheste United Kingdom: John Wiley \& Sons Ltd, 2008.

[34] F. W. Grover, Inductance Calculations: Working Formulas and Tables. Mineola, New York: Dover Publications, 1946.

[35] P. D. Marc C. Thompson, "Inductance Calculation Techniques - Part II: Approximations and Handbook Methods," 1999.

[36] R. Dengler, "Self inductance of a wire loop as a curve integral." pp. 1-14, 04-Apr-2012.

[37] R.-B. Wu, C.-N. Kuo, and K. K. Chang, "Inductance and resistance computations for three-dimensional multiconductor interconnection structures," IEEE Trans. Microw. Theory Tech., vol. 40, no. 2, pp. 263-271, 1992.

[38] V. G. Sapogin and N. N. Prokopenko, "Running inductance of cylindrical conductors with axial current density," in 2014 International Conference on Signals and Electronic Systems (ICSES), 2014, pp. 1-4.

[39] J. A. Camara and PE, Power Reference Manual for the Electrical and Computer PE Exam (EPRM). Belmont, CA, CA: Professional Publications, Inc., 2010.

[40] E. . Rosa, "The self and mutual inductances of linear conductors," Bulletin of the Bureau of Standards, 1907. [Online]. Available: https://archive.org/details/formulae5113219089393rosa. [Accessed: 07-Jul-2015].

[41] A. Russell, "The Effective Resistance and Inductance of a Concentric Main, and Methods of Computing the Ber and Bei and Allied Functions," Proc. Phys. Soc. London, vol. 21, no. 1, pp. 581-614, Dec. 1907.

[42] D. Meeker, "Finite Element Method Magnetics," 2014. [Online]. Available: http://www.femm.info. 